$10-15-2012$

\title{
Synthesis of Di- and Trisubstituted Azulenes Using a Danheiser Annulation as the Key Step: An Advanced Organic Laboratory Experiment
}

Rebecca M. Thomas

Kevin M. Shea

Smith College, kshea@smith.edu

Follow this and additional works at: https://scholarworks.smith.edu/chm_facpubs

Part of the Chemistry Commons

\section{Recommended Citation}

Thomas, Rebecca M. and Shea, Kevin M., "Synthesis of Di- and Trisubstituted Azulenes Using a Danheiser Annulation as the Key Step: An Advanced Organic Laboratory Experiment" (2012). Chemistry: Faculty Publications, Smith College, Northampton, MA. https://scholarworks.smith.edu/chm_facpubs/13 


\title{
Synthesis of Di- and Trisubstituted Azulenes Using a Danheiser Annulation as the Key Step: An Advanced Organic Laboratory Experiment
}

\author{
Rebecca M. Thomas and Kevin M. Shea* \\ Department of Chemistry, Smith College, Northampton, Massachusetts 01063, United States
}

\section{ABSTRACT:}

This three-week experiment provides students with an inquiry-based approach focused on learning traditional skills like primary literature interpretation, reaction design, flash column chromatography, and NMR analysis. Additionally, students address higher order concepts like the origin of azulene's blue color, the mechanism of the Danheiser annulation (step 1), identification of an unknown reaction byproduct, and rationalization of a selective electrophilic aromatic substitution reaction (step 2). Students are initially drawn in by the fascinating color of azulene and remain engaged by the intriguing questions that naturally occur

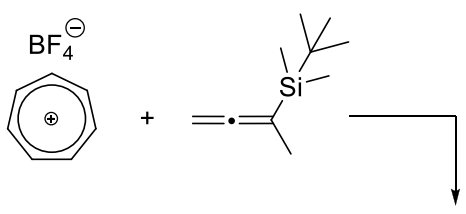
during this investigation.

\section{KEYWORDS:}

Upper-Division Undergraduate, Organic Chemistry, Inquiry-Based, Aromatic Compounds, Carbocations, Chromatography, Electrophilic Substitution, Mechanisms of Reactions, NMR Spectroscopy, Synthesis

Readers of the Journal are familiar with the components of a successful advanced organic laboratory experiment. ${ }^{1}$ It should be reliable, thought-provoking, and discovery-based. It should help students learn concepts and techniques not encountered in introductory organic lab classes. Furthermore, the experiment should help prepare students for the unexpected challenges that occur in research laboratories.

In order to provide students with such an experience, an experiment was developed based on Danheiser's annulation strategy for the synthesis of substituted azulenes ${ }^{2}$ that uses flash chromatography and NMR interpretation techniques. The overall reaction involves the combination of tropylium tetrafluoroborate (1), allenylsilane $\mathbf{2}$, and a base to yield disubstituted 
azulene $\mathbf{3}$ as seen in Scheme 1. Our primary goal for this experiment is to introduce students to a research-like environment by having them repeat two reactions from the literature. At the conclusion of this three-week experience, students should be more comfortable interpreting and adapting literature procedures, purifying mixtures by flash chromatography, running and analyzing ${ }^{1} \mathrm{H}$ NMR spectra, isolating and identifying multiple reaction products, understanding complicated reaction mechanisms, and reporting their results in a journal format. Our analysis and conclusions from this experiment are based on a total of 85 students completing this lab working in teams of two and three from 2008-2012.

\section{Scheme 1. Danheiser Annulation to Yield a Disubstituted Azulene}

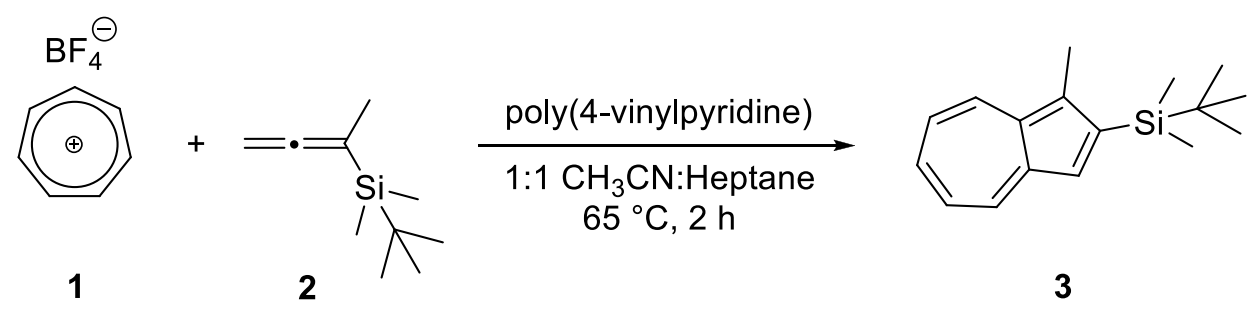

This particular reaction was appealing because of azulene's brilliant appearance and the practical advantage of students running their first flash column on a colored molecule. The color of the product also inspired an outreach program to use a similar Danheiser annulation. ${ }^{3}$ Numerous other papers in the Journal focus on the electronic and spectroscopic properties of azulene and substituted analogs ${ }^{4}$, as well as the syntheses of azulene ${ }^{5}$ and a trisubstituted azulene ${ }^{6}$.

\section{EXPERIMENT, RESULTS, AND DISCUSSION}

Students completed the reaction sequence in Scheme 2 over three weeks. ${ }^{7}$ Week one involved the synthesis of disubstituted azulene $\mathbf{3}$ (following Danheiser's procedure ${ }^{2 \mathrm{a}}$ ), week two was purification and analysis of $\mathbf{3}$, and week three was the synthesis, purification, and analysis of trisubstituted azulene 4 (following Hafner's procedure ${ }^{8}$ ).

\section{Scheme 2. Synthesis of a Trisubstituted Azulene}

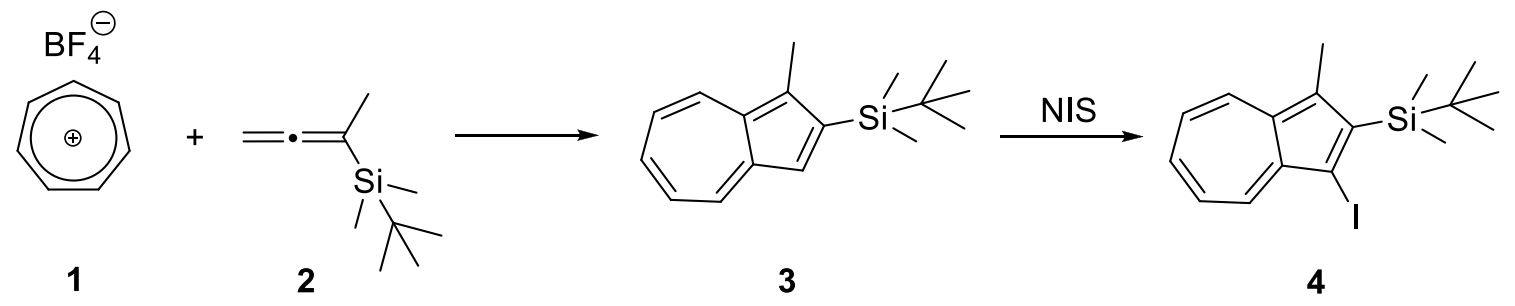


Prior to the initial recitation meeting, students read the lab handout (see Supporting Information) and the Danheiser ${ }^{2 a}$ and Hafner ${ }^{8 a}$ papers. Class began with student questions about Danheiser's literature procedure. This stimulated much discussion since the paper provided the following experimental details: "reaction was carried out with 3 equiv $\mathrm{TpBF}_{4}$ and poly(4vinylpyridine) $\left(123 \mathrm{mg} / \mathrm{mmol}\right.$ of $\left.\mathrm{TpBF}_{4}\right)$ in $1: 1 \mathrm{CH}_{3} \mathrm{CN}$-heptane at $65^{\circ} \mathrm{C}$ for $2 \mathrm{~h}$ ". This was a stark change from the detailed instructions provided in introductory labs. Consequently, discussion reinforced the construction of a mole table and aqueous work up conditions. Students also thought about the mechanism for this complicated reaction. After running the reaction, students were thrilled to have formed an unexpectedly blue product. This sparked their curiosity, and they began to wonder why a compound with azulene's structure is blue.

Students analyzed the ${ }^{1} \mathrm{H}$ NMR of allene $2,{ }^{9}$ predicted the ${ }^{1} \mathrm{H}$ NMR of product $\mathbf{3}$, and analyzed their crude reaction product by TLC and ${ }^{1} \mathrm{H}$ NMR. Since most students have never seen an allene before, the ${ }^{1} \mathrm{H}$ NMR of compound 2 was surprising (Figure 1). They were often convinced that the NMR was incorrect because there was "clearly an ethyl group in this molecule". To help them understand this spectrum, students were encouraged to make a molecular model of the allene, and the concept of long range splitting across $\pi$ bonds was introduced. ${ }^{10}$

Figure 1. Allene ${ }^{1}$ H NMR Assignment ${ }^{11}$

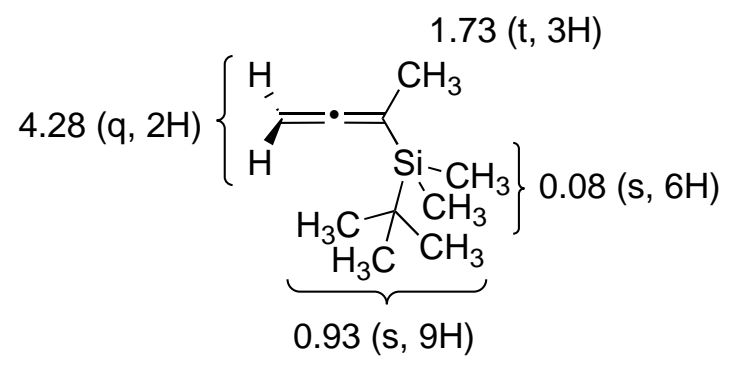

The TLC analysis of the crude product was also troubling. Eluting with hexanes, students saw two blue spots with similar Rf values. They concluded that more than one azulene product formed which was confirmed by their ${ }^{1} \mathrm{H}$ NMR analysis. This spectrum contained an unexpectedly large number of peaks in the aromatic region and several peaks in the vinyl region. Students noted that this is not surprising since the literature yield for the reaction is $22 \%^{2 a}$. 
Figure 2. Flash Column Purification of Reaction One

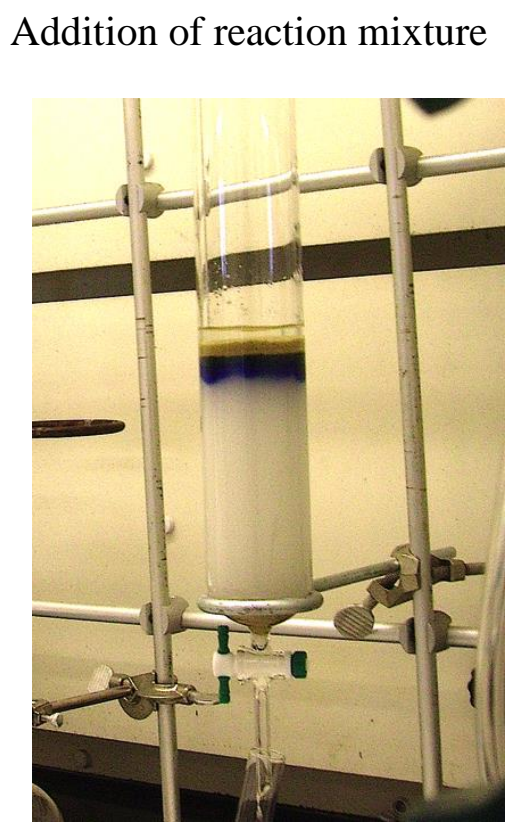

Initial separation

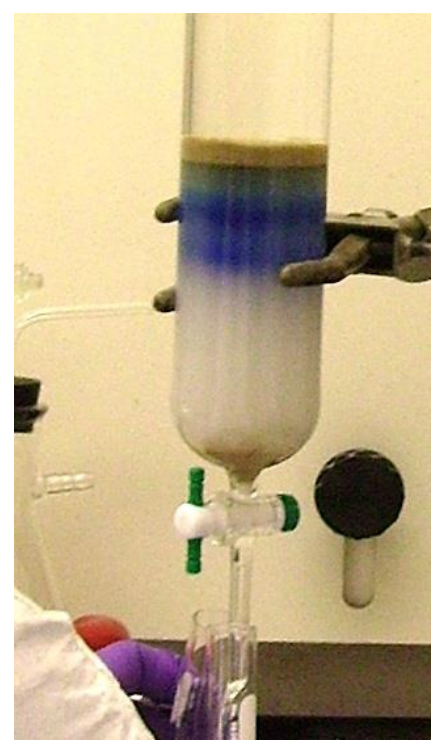

Complete separation

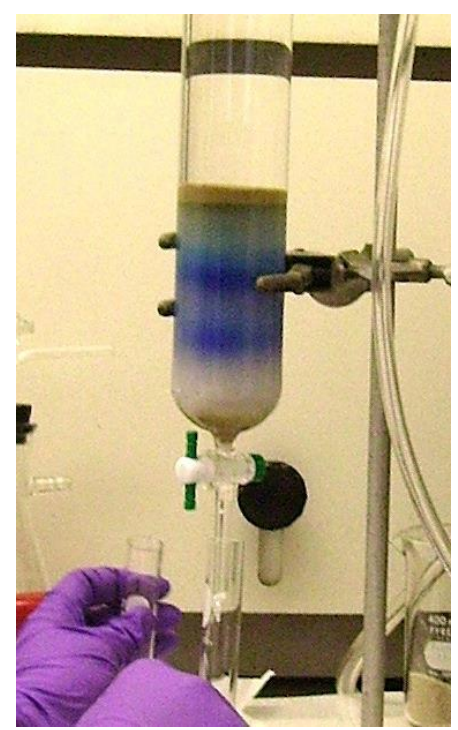

Students purified their reaction mixtures by flash chromatography and separated the two azulene fractions (Figure 2). Then they determined which was their desired product for use in the subsequent iodination reaction. ${ }^{1} \mathrm{H}$ NMR analysis of each fraction demonstrated that the higher Rf compound was disubstituted azulene 3 (Figure 3). They were unsure of the structure of the other molecule (Figure 4) and had no explanation for how another azulene formed.

Figure 3. Partial ${ }^{1}$ H NMR Spectrum and Assignments for Disubstituted Azulene 3

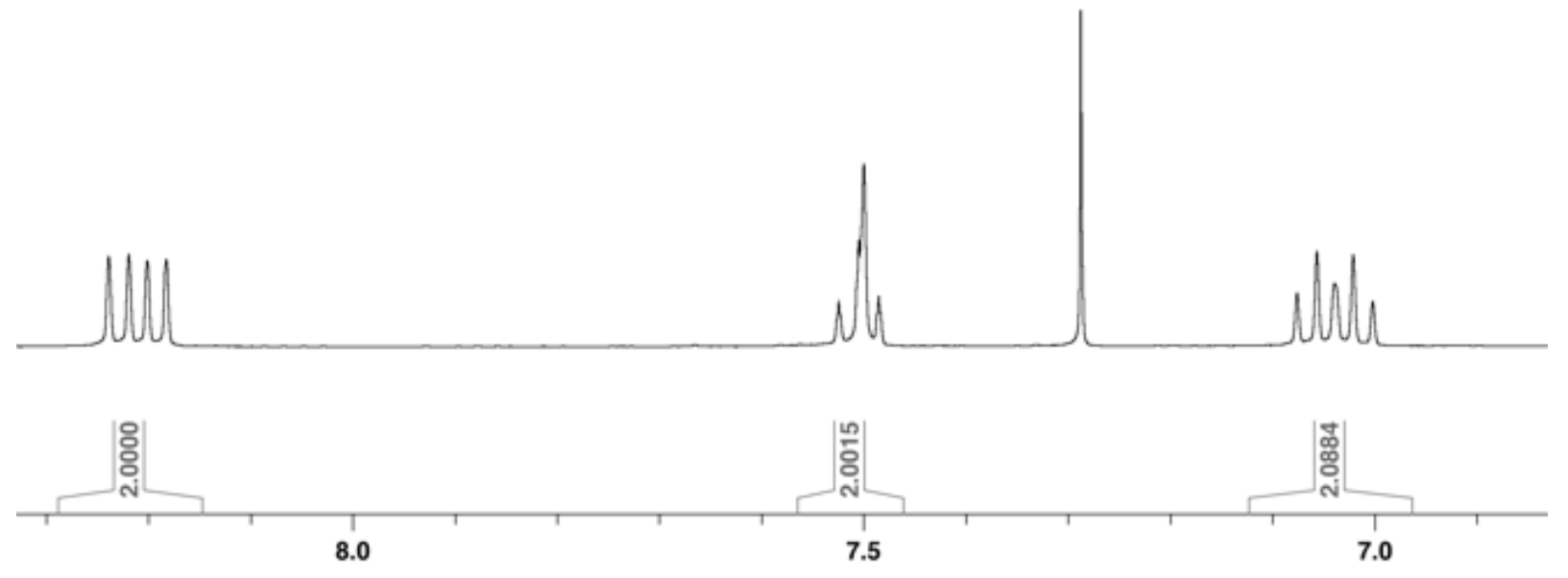




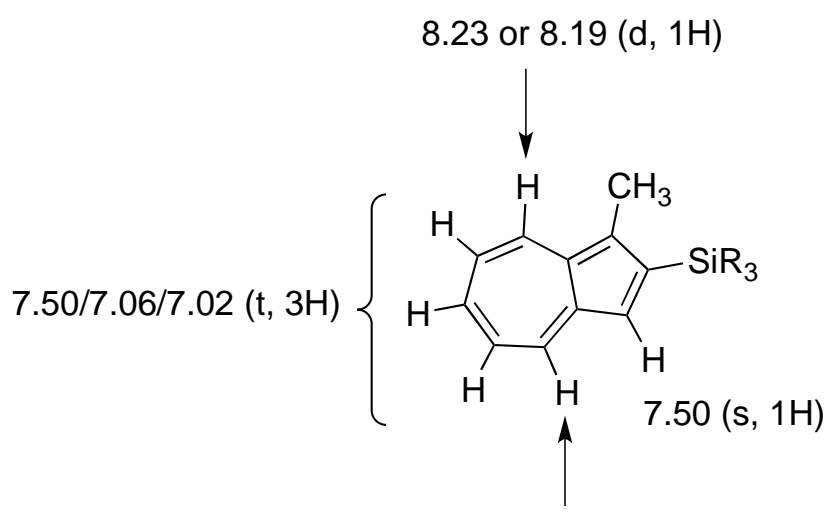

8.23 or $8.19(\mathrm{~d}, 1 \mathrm{H})$

3

Figure 4. Partial ${ }^{1} \mathrm{H}$ NMR Spectrum for Unknown Azulene Byproduct

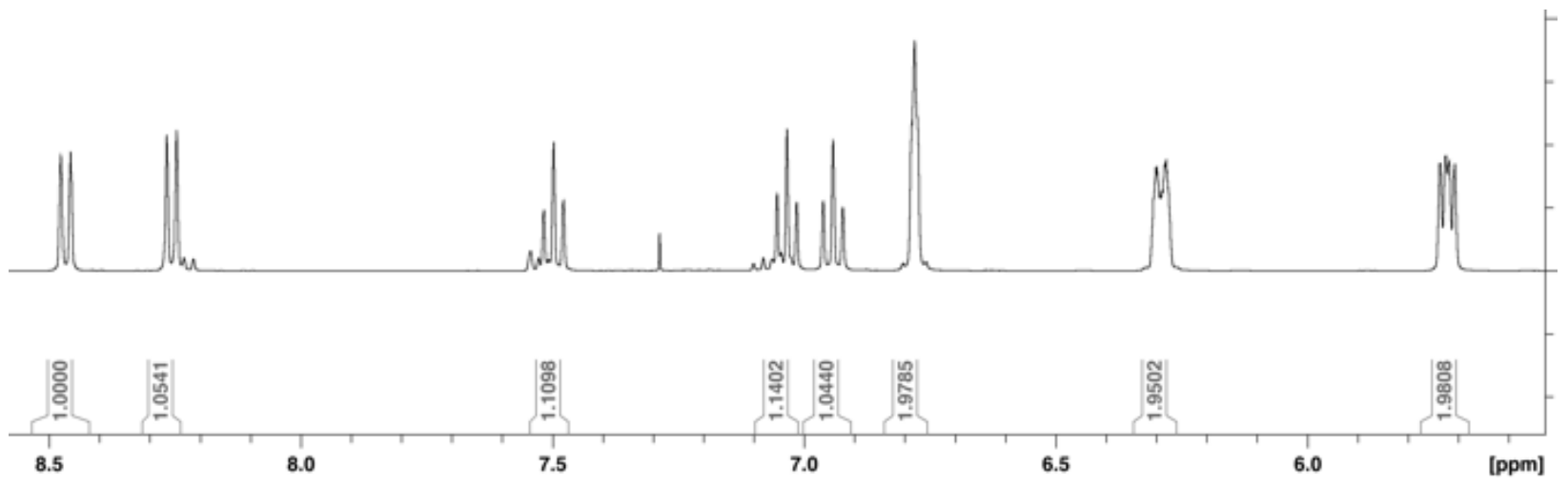

The iodination and purification proceeded uneventfully to the desired trisubstituted azulene, but this transformation led to another question. Why was this electrophilic aromatic substitution reaction ${ }^{12}$ completely selective for the position adjacent to the bulky $t$ butyldimethylsilyl group?

Once azulene 4 was purified, students began to write their paper describing this experiment. ${ }^{13}$ The key questions that remained were 1) Why is azulene blue? 2) What is the mechanism for formation of azulene 3? 3) What is the structure of the undesired azulene byproduct? 4) What is the mechanism for formation of this byproduct? 5) Why did the iodination reaction occur in the five-membered ring?

Students were challenged to "think like chemists" to answer these questions, and the instructors were thrilled when students used the chemical literature to generate outstanding answers. Regarding why azulene is blue, students struggled to understand the accepted explanation of a small HOMO-LUMO gap resulting from differences between alternant (naphthalene) and nonalternant (azulene) aromatic hydrocarbons. ${ }^{14}$ 
The mechanism for the formation of azulene $\mathbf{3}$ is outlined in Danheiser's paper ${ }^{2 a}$; however, it skips over several steps that are critical to understand this reaction. The complete mechanism is depicted in Scheme 3 and involves attack of tropylium (1) by allene $\mathbf{2}$ to yield cation 5 which is stabilized by the silicon $\beta$-effect. ${ }^{15}$ A silyl shift provides isomeric cation 6 which cyclizes to resonance stabilized cation $\mathbf{7}$ and furnishes dihydroazulene $\mathbf{8}$ after deprotonation. Most students struggled to explain conversion of $\mathbf{8}$ into $\mathbf{1 1}$ and called this a deprotonation facilitated by the pyridine base. This was a reaction they had probably never seen before, a hydride transfer from $\mathbf{8}$ to a second equivalent of tropylium to yield cycloheptatriene (9) and resonance stabilized cation 10. This unexpected step can be rationalized by recognition that $\mathbf{1 0}$ is better represented by 11, an aromatic, substituted tropylium ion. A final deprotonation reaction yields azulene 3 .

\section{Scheme 3. Mechanism for Formation of Azulene 3}

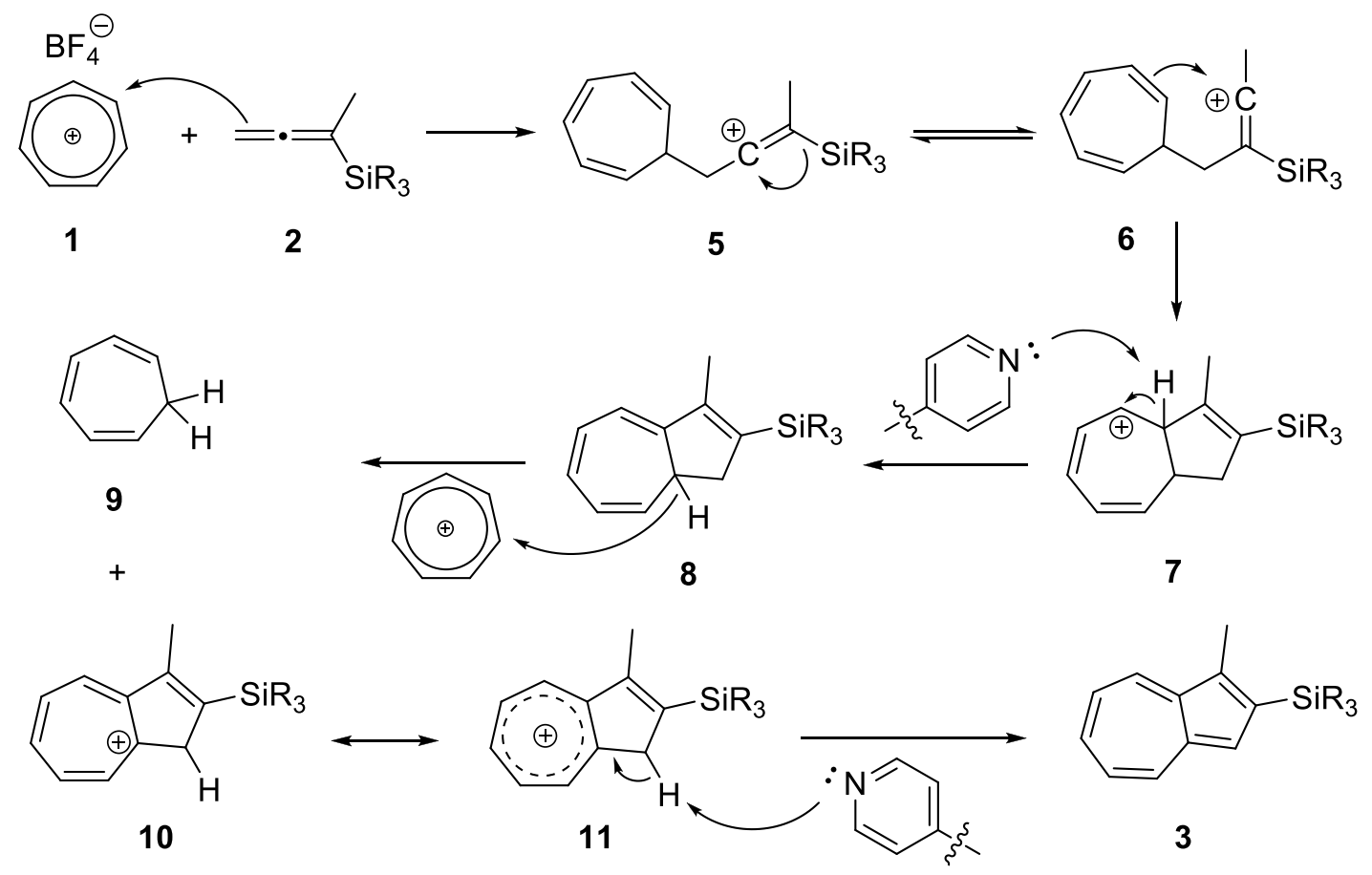

The structure of the byproduct and mechanism for its formation were the most difficult questions to answer. However, students who read the Danheiser paper ${ }^{2 a}$ very carefully were the first ones to obtain the solution. Danheiser states that "reactions employing allenylsilanes lacking C-3 substituents proceed in diminished yield due to partial destruction of annulation product initiated by the electrophilic attack of tropylium cation at C-3 of the azulene." Thus, the mystery is solved. The undesired azulene product is a trisubstituted azulene with a cycloheptatrienyl substituent at the C-3 position (14) (see Figures 4 and 5) resulting from an electrophilic aromatic substitution reaction (Scheme 4) after the annulation step. ${ }^{16}$ 
Scheme 4. Structure and Mechanism for Formation of Trisubstituted Azulene 14

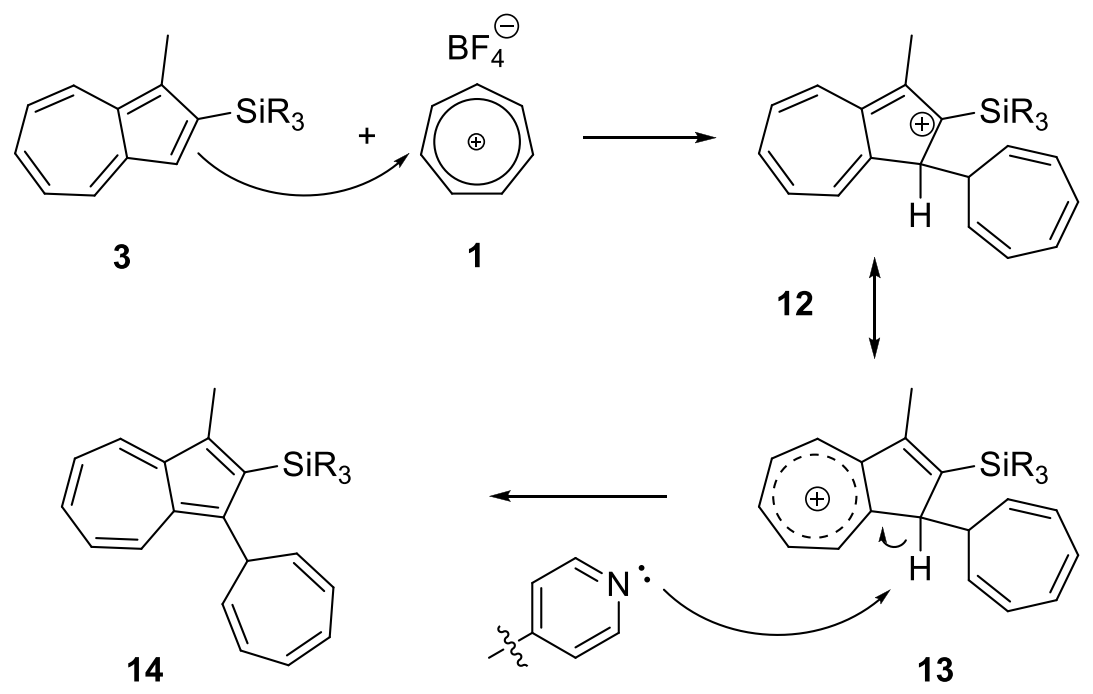

Figure 5. Partial ${ }^{1} \mathrm{H}$ NMR Assignments for Trisubstituted Azulene 14

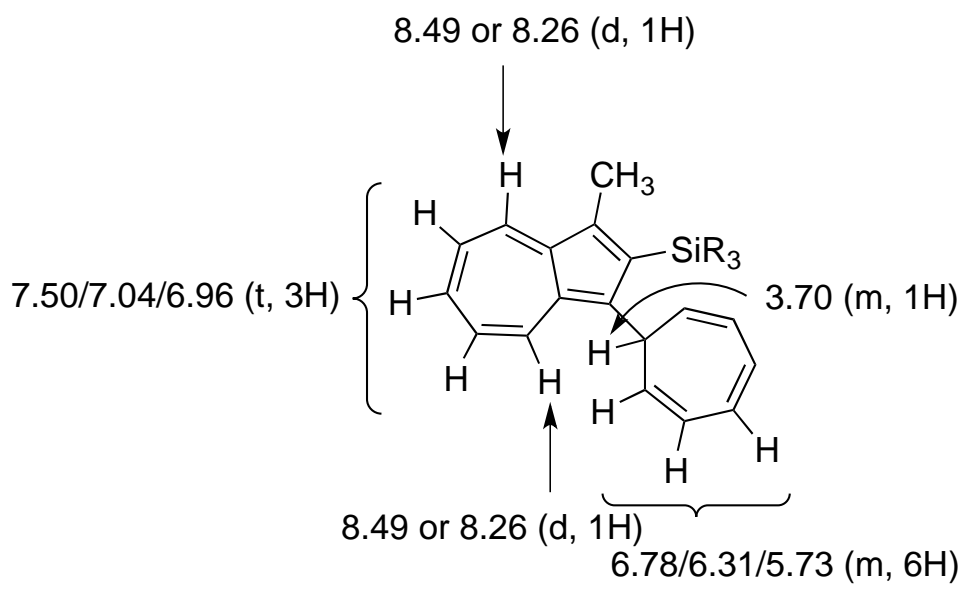

14

Students completed their table of reaction yields for both steps which included the production of disbustituted azulene $\mathbf{3}$ and trisubstituted azulenes $\mathbf{1 4}$ and $\mathbf{4}$. As shown in Table 1, yields for the first step were low, while the second step worked well. 
Table 1. Student Yields for Reactions One and Two

\begin{tabular}{ccc}
\hline $\begin{array}{c}\text { Yield of Disubstituted } \\
\text { Azulene } \mathbf{3}\end{array}$ & $\begin{array}{c}\text { Yield of Undesired } \\
\text { Trisubstituted Azulene } \mathbf{1 4}\end{array}$ & $\begin{array}{c}\text { Yield of Trisubstituted } \\
\text { Iodoazulene } \mathbf{4}\end{array}$ \\
\hline $7-25 \%$ & $10-29 \%$ & $66-80 \%$ \\
\hline
\end{tabular}

The reactivity of disubstituted azulene $\mathbf{3}$ can be explained by learning about polarized aromatic molecules and aromaticity of cationic reaction intermediates. Students were comfortable thinking about the reactivity of alternant aromatics like benzene and could predict major and minor products for reactions of substituted benzenes based on the stabilities of cationic intermediates. While the same strategy must be employed to explain the outcome of the second reaction (and the formation of byproduct 14), it was critical for students to gain a deeper understanding of Hückel's rule and its application to cations like tropylium. ${ }^{17}$ It was then straightforward to analyze all of the possible cationic intermediates resulting from reaction at the unsubstituted positions in disubstituted azulene 3 (Scheme 5). Reaction in the seven-membered ring would lead to cationic intermediates like $\mathbf{1 8}$ that have antiaromatic character resulting from delocalization of the cation into the five-membered ring, while reaction in the five-membered ring leads to cationic intermediate $\mathbf{1 6}$ having aromatic character resulting from delocalizaton of the cation into the seven-membered ring. ${ }^{18}$

\section{Scheme 5. Electrophilic Aromatic Substitution Mechanisms for Reaction of Azulene 3}

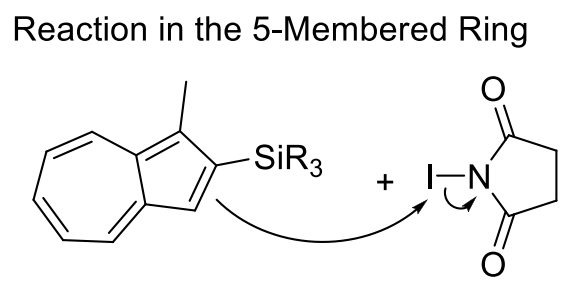

3

Reaction in the 7-Membered Ring

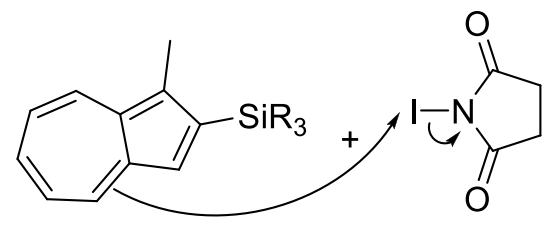

3

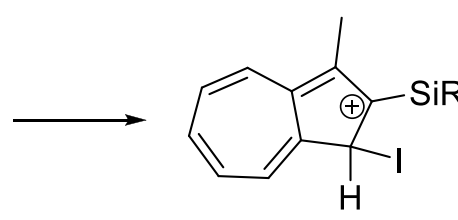

15

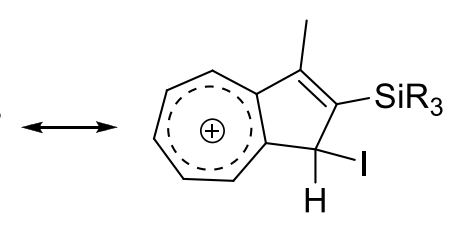

16

stable aromatic cation

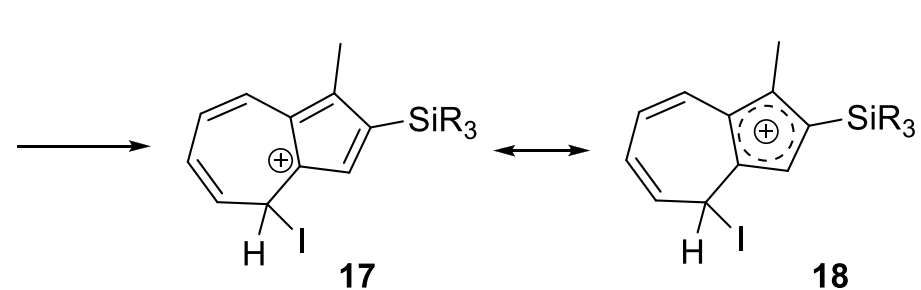

unstable antiaromatic cation

CONCLUSION 
This three-week experiment fostered student learning and prepared students for the challenges they will face in a research lab. With minimal guidance from their instructors, students ran two reactions, purified the reaction products by flash chromatography, and analyzed their results by ${ }^{1} \mathrm{H}$ NMR. They learned critical skills associated with repeating reactions from the primary literature, identifying unexpected reaction byproducts, thinking critically about reaction mechanisms, and understanding regioselectivity in electrophilic aromatic substitution reactions. Finally, students presented their results and analysis just like professional chemists using the Organic Letters format and template.

Many of these challenges occur routinely in the research lab, and this experiment helped students develop the skills and confidence to deal with unexpected situations that arise while conducting independent investigations. Students were able to demonstrate their mastery of our objectives during the independent project portion of our course which involved repeating several reactions from the literature that students chose in consultation with their instructors. They were much more comfortable and efficient with their experimental and analysis skills and were able to more critically evaluate results. Furthermore, students who completed the course where this lab was taught had a much smoother and productive transition into several of the research labs in our department than students who did not complete this class.

\section{HAZARDS}

Acetonitrile, heptane, and petroleum ether are flammable. Tropylium tetrafluorborate causes skin and eye burns and is harmful if inhaled. Poly(4-vinylpyridine) and Niodosuccinimide are irritants. Chloroform- $d$ is carcinogenic in cases of chronic exposure. All experiments were conducted in fume hoods with students wearing eye protection, lab coats, and nitrile gloves.

\section{ASSOCIATED CONTENT}

\section{Supporting Information}

Student handout; instructor notes; ${ }^{1} \mathrm{H}$ NMR spectra of compounds 2, 3, 4, and 14; COSY spectra of compounds $\mathbf{3}, \mathbf{4}$, and 14; mass spectra of $\mathbf{3}, \mathbf{4}$, and $\mathbf{1 4}$; procedures for the synthesis of $\mathbf{2}$, 3, 4, and 14; and sample student paper. This material is available via the Internet at http://pubs.acs.org.

\section{AUTHOR INFORMATION}

Corresponding Author

*Email: kshea@smith.edu

\section{ACKNOWLEDGMENT}

We acknowledge all of the students from CHM 326: Synthesis and Structural Analysis from 2008-2012 and the students in KMS's research lab who synthesized allene 2. We are grateful to the Smith College Chemistry Department for financial support. 
(1) Schepmann, H. G.; Mynderse, M. J. Chem. Educ. 2010, 87, 721-723 and references therein. (2) a) Becker, D. A.; Danheiser, R. L. J. Am. Chem. Soc. 1989, 111, 389-391. b) Hafner, K.; Stephan, A.; Bernhard, C. Liebigs Ann. Chem. 1961, 650, 42-62.

(3) Nowick, J. S.; Brisbois, R. G. J. Chem. Educ. 1989, 66, 668.

(4) Liu, R. S. H. J. Chem. Educ. 2002, 79, 183-185 and references therein.

(5) Lemal, D. M.; Goldman, G. D. J. Chem. Educ. 1988, 65, 923-925.

(6) Garst, M. E.; Hochlowski, J. H.; Douglass, J. G., III; Sasse, S. J. Chem. Educ. 1983, 60, 510511.

(7) Our class consists of one four-hour lab period and two 80-minutes recitation sections per week.

(8) a) Fabian, K. H. H.; Elwahy, A. H. M.; Hafner, K. Eur. J. Org. Chem. 2006, 791-802. b) Hafner, K.; Patzelt, H.; Kaiser, H. Liebigs Ann. Chem. 1962, 656, 24-33.

(9) Allene $\mathbf{2}$ is not commercially available and can be most easily synthesized in three steps starting with propargyl alcohol according to a) Bishop, B. C.; Cottrell, I. F.; Hands, D. Synthesis 1997, 11, 1315-1320. b) Danheiser, R. L.; Stoner, E. J.; Koyama, H.; Yamashita, D. S.; Klade, C. A. J. Am. Chem. Soc. 1989, 111, 4407-4413. This synthesis is an excellent starter project for beginning organic synthesis research students. It should be noted that allene $\mathbf{2}$ was approximately $90 \%$ pure by ${ }^{1} \mathrm{H}$ NMR. The allene can be purified further according to reference $9 \mathrm{~b}$, but this was unnecessary for our purposes. Full details are available in the supporting information of this paper.

(10) It is also possible to rule out the presence of an ethyl group by analysis of the magnitude of the coupling constants. Ethyl groups couple with $J$ values around $8 \mathrm{~Hz}$ while the long range allene protons-to-methyl group coupling we observe is around $3 \mathrm{~Hz}$. For a reference, see Lambert, J. B.; Mazzola, E. P. Nuclear Magnetic Resonance Spectroscopy; Pearson: Upper Saddle River, NJ, 2004, 98-130.

(11) All of the data, including the spectra, in the manuscript and the Supporting Information are student generated.

(12) In some years, students were encouraged to explore other electrophilic aromatic substitution reactions instead of this iodination reaction. However, exploration of chlorination, bromination, acetylation, and sulfination reactions proved unexpectedly challenging due to complications including unstable reaction products, sluggish reaction rates, and difficult purifications.

(13) Again sticking with the theme of preparing students for the research lab, there are no "lab reports" in this course. At the conclusion of each experiment, students write a "paper" using the Organic Letters template available for download at the Organic Letters website. For an example of an actual student paper for this experiment, please see the supporting information.

(14) The following are a list of references identified and used by the students during class discussions: a) Ref. 4 b) Selco, J. I.; Brooks, T.; Chang, M.; Trieu, M. T.; McDonald, J. K.; McManus, S. P. J. Org. Chem. 1994, 59, 429-433. c) Michl, J.; Thulstrup, E. W. Tetrahedron 1976, 32, 205-209. d) Mann, D. E.; Platt, J. R.; Klevens, H. B. J. Chem. Phys. 1949, 17, 481-484. 
(15) Clayden, J.; Greeves, N.; Warren, S.; Wothers, P. Organic Chemistry; Oxford University Press: New York, 2001; pp 1291-2.

(16) Attempts to suppress formation of this undesired product by variation in reaction times and temperatures as well as reduction in amounts of tropylium tetrafluoroborate have never resulted in increased yields of the desired disubstituted azulene product. In general, students with more lab experience, especially with flash chromatography, obtain higher yields of the two azulene products.

(17) Jones, M., Jr.; Fleming, S. A. Organic Chemistry, $4^{\text {th }}$ ed.; Norton: New York, 2010, 582-595. (18) We discuss how the reactions to form both trisubstituted azulenes $\mathbf{4}$ and $\mathbf{1 4}$ proceed via similar electrophilic aromatic substitution mechanisms. 